\title{
Avaliação do ruído ambiental em uma mineradora
}

Marluce Teixeira Andrade Queiroz marluce.queiroz@bol.com.br Centro Universitário do Leste de Minas Gerais (UNILESTE), Coronel Fabriciano, Minas Gerais, Brasil

Luiz Otávio Caldeira Brant luizbrant@yahoo.com.br

Centro Universitário do Leste de Minas Gerais (UNILESTE), Coronel Fabriciano, Minas Gerais, Brasil

\section{Carolina Andrade Queiroz}

carolanq@yahoo.com.br

Instituto Nacional de Seguridade Social (INSS), Rio de Janeiro, Rio de Janeiro, Brasil

\section{Nathielly Rangel Teodoro Batista} thielly_13@hotmail.com

Centro Universitário do Leste de Minas Gerais (UNILESTE), Coronel Fabriciano, Minas Gerais, Brasil

Felipe Andrade Queiroz felipeandradeq@yahoo.com.b Centro Universitário do Leste de Minas Gerais (UNILESTE), Coronel Fabriciano, Minas Gerais, Brasil

\begin{abstract}
RESUMO
O sincronismo entre as atividades industriais e a gestão empresarial são condições essenciais para se alcançar o controle econômico com minoração dos fatores desestabilizadores da vida humana e repercussões adversas nos demais componentes bióticos. Nesse sentido, o atendimento das necessidades de segurança e saúde dos trabalhadores e da população estão intrinsecamente relacionados. Entende-se aí a relevância quanto ao monitoramento dos riscos ambientais. Por essa razão, buscou-se identificar os impactos relacionados ao ruído produzidos por um empreendimento do ramo de mineração. Os resultados então obtidos foram comparados com o disposto na ABNT 10151/2000 e as recomendações da norma ISO/1996. Verificou-se que as condições de trabalho no ambiente interno da mina, apresentaram dinâmica de operação padrão, porém, com limites de condições de audição desfavoráveis (ambientes ruidosos). Admitem-se, assim, medidas objetivas de controle para os trabalhadores, tais como: enclausuramentos, encamisamentos, barreiras, inserção de atenuadores. Em ambiente externo da mina, o ruído gerado ficou abaixo do Nível de Conforto Auditivo (NCA) em todos os períodos avaliados. Entretanto, constatou-se que os moradores nas proximidades do empreendimento apresentavam queixas pertinentes à poluição sonora. Nessa perspectiva, recomenda-se aproveitamento máximo dos obstáculos naturais investindo na manutenção da vegetação nativa.
\end{abstract}

PALAVRAS-CHAVE: Mineração. Ruído Ambiental. Segurança do Trabalho. Mitigação. 


\section{INTRODUÇÃO}

Lobo et al (2015) afirmam que a poluição sonora é um problema com reflexos negativos que são dectectados em diversos empreendimentos econômicos ao nível da realidade global. Em outro ângulo, Caixeta et al. (2016) ponderam que existem diversas estratégias para contenção do risco, entretanto, a efetiva mitigação depende de vários fatores, tal como, seleção adequada das soluções tecnológicas e disponibilidade financeira. Mas, mesmo em locais onde uma grande quantidade de recursos foi aplicada para regular ou reduzir o ruído ambiental, o problema persiste ou até se agravou. Por exemplo, recursos extensivos foram aplicados no sentido de redução do ruído produzido pelo tráfego de automóvel na fonte. De fato, hoje, os veículos são muito mais silenciosos do que antes. No entanto, o volume de tráfego aumentou de tal ordem que os avanços conseguidos devido às novas gerações de automóveis foram ultrapassados pelo ruído adicional produzido pelo dramático aumento do número de veículos em circulação (GOLDNER et al., 2014).

Nas grandes cidades, o trânsito é o grande causador dos ruídos. As características dos veículos barulhentos são o escape furado ou enferrujado, as alterações no silenciador ou no tubo de escape, os maus hábitos ao conduzir, acelerações, travagens bruscas e o uso excessivo da buzina. Mas, como sabemos o trânsito não é o único causador de ruídos. Outros exemplos são as construções e obras que se espalham pela cidade, locais de grande afluência pública (SANTOS e DE FREITAS, 2014).

Dentre as atividades industriais responsáveis pelo incremento da poluição sonora destaca-se a mineração. Trata-se de setor essencial para economia do país, que contribui de forma decisiva para o bem estar e a melhoria da qualidade de vida das gerações presentes e futuras, sendo fundamental para o desenvolvimento de uma sociedade equânime. O reconhecimento e controle dos impactos que essa atividade provoca ao meio ambiente permitem que o empreendimento seja operado com responsabilidade social, agregando valoração ao atender os preceitos do desenvolvimento sustentável (SILVA e LASCHEFSKI, 2012).

Segundo o Ministério de Minas e Energia (MME) a mineração é responsável por impactos ambientais diversos e apresentam-se em diversas escalas: desde problemas locais específicos até alterações biológicas, geomorfológicas, hídricas e atmosféricas de grandes proporções. A indústria da mineração ocupa grandes áreas para garantir a extração da matéria-prima e desse modo, ocorre à supressão de vegetação ou impedimento de sua regeneração (BENTO COELHO, 2009). Em diversas ocasiões, a camada superficial com abundância em matéria orgânica é removida, e os solos expostos são mais suscetíveis aos processos erosivos e podem acarretar em assoreamento dos cursos de águas (CANDIA et al, 2010).

Sethurajan et al (2016) reforçam que a mineração contribui também para a poluição do ar através da emissão de particulados suspensos nas fases de extração, beneficiamento e transporte. Os pesquisadores enfatizam a ocorrência de emissão de gases derivados da queima de combustíveis, tal como, gás carbônico, óxidos de enxofre e hidrocarbonetos não queimados. Di Giulio et al. (2015) relatam que outros impactos ao meio ambiente são a poluição sonora, 
sobrepressão acústica e vibrações no solo associados à operação de equipamentos e explosões

Portanto, identificar os problemas causados em função da mineração e a minimização de seus efeitos é de grande necessidade para garantir a preservação dos ambientes naturais. Nesse contexto, atentou-se quanto ao nível de poluição sonora exercido por empresa daquele segmento econômico localizada no município de Brumadinho, Minas Gerais, Brasil. O estudo de caso norteou-se para mensuração do risco do ruído conforme as exigências da ABNT 10151/00 e proposição das medidas de contenção. Entende-se que a divulgação destes resultados pode contribuir para a sistematização de programas de gestão em segurança e saúde em situações similares.

\section{REFERENCIAL TEÓRICO}

\section{RUÍDO}

O ruído é um risco físico com características próprias que podem incomodar o ser humano em função das suas características, tal como, timbre e nível sonoro (Kähari et al, 2003). Em função das suas características são detectados diferentes tipos de ruídos e desse modo requerem diferentes estratégias para a sua medição e mitigação (Rabinowitz et al., 2007).

Os fatores mais importantes que afetam a propagação sonora no ambiente são o tipo de fonte, a distância da fonte, a absorção atmosférica, os obstáculos, tais como barreiras e edifícios, absorção do solo, velocidade do vento, edificações, dentre outros (SILVA, 2007).

As operações de máquinas e equipamentos em mineradoras podem contribuir com a poluição sonora no seu entorno. Esse agente físico pode implicar em grave desconforto para a população exposta podendo ocasionar uma grande sensação de incomodo e irritabilidade (HARGER e BARBOSA BRANCO, 2004). O risco ambiental pode se apresentar como ruído contínuo produzido por máquinas que funcionam sem interrupção ou como ruído de impacto ou impulsivo provocado por explosões, sendo breve e abrupto (SUTER, 2002). Especificamente em relação ao ruído de impacto é comum a ocorrência da surdez temporária no indivíduo exposto. Melnick (2000), citado por Caixeta et al (2016), afirma que o tempo de recuperação da mudança do limiar auditivo, ainda é completamente indefinido, podendo ser de poucos minutos a diversas semanas. Esta mudança pode variar de uma sensibilidade auditiva de alguns poucos decibéis, insignificantes, em uma faixa estreita de frequências, até as alterações que tornam o ouvido temporariamente surdo, sendo, nesse caso uma sensação de desconforto transitória (Mahendra e Sridhar, 2008).

Dela Coleta (1989) informa que os efeitos adversos do ruído proveniente das operações industriais podem extrapolar o ambiente interno e desse modo atingir negativamente a população não ocupacional. O pesquisador descreve a existência da relação intrínseca entre o ruído ambiente e os efeitos na saúde humana através de alterações em mecanismos fisiológicos. Santos (1994) relata que a exposição ao ruído ambiente pode levar a perturbações do sono e atividades diárias, ao aborrecimento e ao stress. Estudos da Organização Mundial 
de Saúde (OMS) mostram que a poluição sonora pode desencadear a produção de hormônios (cortisol, adrenalina e noradrenalina), o que pode levar a uma variedade de efeitos intermediários, incluindo o aumento da pressão arterial. Ao longo de um período prolongado de exposição estes efeitos podem por sua vez aumentar o risco de doenças cardiovasculares e distúrbios psiquiátricos (BENTO COELHO, 2009). O grau de ruído leva à perturbação dos indivíduos, irritabilidade e stress dependendo em parte das características psicofisiológicas do indivíduo (WHO, 2009).

\section{AVALIAÇÃO AMBIENTAL DO RUÍDO}

A percepção auditiva do indíviuo resulta da relação entre a intensidade e a frequência do som, o qual nos dá o nível de pressão sonora expresso em decibéis (BELLUSCl, 2012). De acordo com Franco (2010) o decibel é definido como razão entre o valor medido e o valor de referência padrão, o qual corresponde a uma mínima variação detectável pelo ouvido humano.

A Norma Técnica Brasileira (NTB) (ABNT, 10151/00) fixa as condições exigíveis para avaliação da aceitabilidade do ruído em comunidades, independente da existência de reclamações. A norma especifica também o método para a medição de ruído, a aplicação de correções nos níveis medidos se o ruído apresentar características especiais e uma comparação dos níveis corrigidos com um critério que leva em conta vários fatores.

O método de avaliação envolve as medições do nível de pressão sonora equivalente (LAeq), em decibéis ponderados em curva de compensação " $A$ ", comumente mensurado em decibéis $(\mathrm{dB}(\mathrm{A}))$, salvo o que consta no item 5.4.2, da ABNT 10151/00 no qual o nível corrigido (LC) para ruído com características impulsivas ou de impacto é determinado pelo valor máximo medido com o medidor de nível de pressão sonora ajustado para resposta rápida (fast), acrescido de $5 \mathrm{~dB}(\mathrm{~A})$.

\section{PROCEDIMENTOS METODOLÓGICOS}

\section{ÁREA DE ESTUDO}

A investigação foi realizada em uma empresa do ramo de mineração, localizada na cidade de Brumadinho, no estado de Minas Gerais, Brasil. O município integra a Região Metropolitana de Belo Horizonte e a população corresponde ao total de trinta e cinco mil e oitenta e cinco (35.085) habitantes conforme o censo do Instituto Brasileiro de Geografia e Estatística (IBGE) em 2012 (IBGE, 2016).

O município de Brumadinho assume posição de destaque em função dos seus recursos hídricos com grandes mananciais, desse modo, juntamente com os municípios vizinhos é responsável por cerca de vinte e cinco por cento $(25 \%)$ da água que abaste a região metropolitana de Belo Horizonte, através dos sistemas Rio Manso e Catarina, operados pela Companhia de Saneamento de Minas Gerais (COPASA) responsável captação, adução, estação de tratamento, reservação, redes de distribuição e ligações domiciliares (COPASA, 2017). 
Mafia et al. (2009) relata que o balanço econômico do município de Brumadinho pode ser atribuído a exploração do minério, água mineral e cachaça artesanal. Os mecanismos de exploração dos recursos naturais (componentes estruturais do ecossistema) implicam em desencadeamento de processo antropogênico com elevada produção de rejeitos com degradação dos componentes bióticos naturais (ANDRADE, 2008).

\section{AVALIAÇÃO DO RUÍDO AMBIENTAL}

Avaliou-se o ruído ambiental gerado nessa mineradora, cuja fonte ruidosa integrava o espaço laboral, sendo a principal fonte poluidora um britador localizado no ponto central da área em estudo (Figura 1). 0 equipamento era utilizado para moagem, uma vez que o mesmo define a granulometria e forma dos fragmentos do minério explorado.

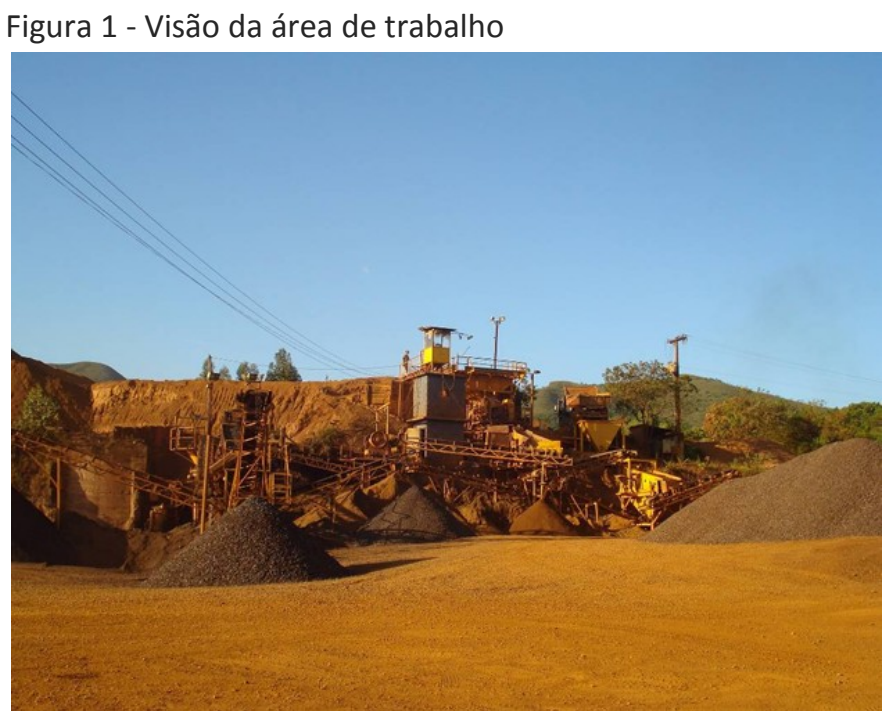

Fonte: Autoria própria (2017).

Utilizou-se para avaliação o dosímetro de ruído da marca Simpson, modelo 897, número de série B004525, certificado pelo INMETRO. Por meio das medições realizadas, observou-se a inexistência de fenômenos da natureza, tais como, trovões, chuvas fortes, em atendimento aos requisitos da ABNT 10151/00. Ainda em consonância com a referida Norma Técnica, as medições foram efetuadas em pontos afastados: aproximadamente $1,2 \mathrm{~m}$ do piso e, pelo menos, $2 \mathrm{~m}$ do limite da propriedade e de quaisquer outras superfícies refletoras, como muros, paredes.

Foram avaliados dois pontos: um no interior da mina e outro no ambiente externo da mina (comunidade). Visando à caracterização adequada do ruído em questão, optou-se pela amostragem em seqüência, conforme preconizado pela ABNT 10151/00. Em cada ponto, foram realizadas 03 (três) amostras pela manhã, 03 (três) amostras pela tarde e 03 (três) amostras pela noite, num total de 09 amostragens, por ponto, com intervalo de tempo de 5 minutos.

Além disso, outro instrumento utilizado para a coleta de dados foi à entrevista individual de vinte moradores em área próxima à mineradora. Esses entrevistados tiveram suas identidades preservadas e informados quanto ao 
objetivo da abordagem sendo reconhecer a percepção dos habitantes da região em relação aos transtornos associados ao ruído. De modo geral, as questões formuladas eram respondidas entre 15 e 20 minutos, sendo investigadas as queixas auditivas, não auditivas, incomodos e sugestões referentes ao controle de ruído no local. Além do formulário contendo os quesitos foi anexado Termo de Consentimento Livre e Esclarecido, onde se esclarecia ao participante os objetivos da pesquisa e este autorizava a utilização dos dados por ele fornecidos. Os dados foram tabulados com a utilização em planilha, e posteriormente foi realizada a filtragem e análise dos dados.

\section{RESULTADOS E DISCUSSÃO}

Os resultados obtidos foram comparados com o Nível de Critério de Avaliação (NCA) de ruídos externos, mensurados em $\mathrm{dB}(\mathrm{A})$, conforme a $\mathrm{ABNT}$ 10151/00. Constatou-se que o empreendimento avaliado enquadrou-se em predominantemente industrial, com $\mathrm{NCA}$ de $70 \mathrm{~dB}(\mathrm{~A})$ e $60 \mathrm{~dB}(\mathrm{~A})$, respectivamente, nos períodos diurno e noturno (Tabela 1 ).

Tabela 1 - Nível de critério de avaliação NCA para ambientes externos, em dB(A)

Tipos de áreas

Área de sítios e fazendas

Área estritamente residencial urbana ou de hospitais ou de escolas

Área mista, predominantemente residencial

Área mista, com vocação comercial e administrativa

Área mista, com vocação recreacional

Área predominantemente industrial
Diurno Noturno

40

35

50

45

55

50

$60 \quad 55$

65 55

70

Fonte: ABNT 10151/2000.

Sendo assim, detectou-se com base no monitoramento ambiental (Figura 2) que, no Ponto 1 (P1), o nível equivalente de ruído foi menor que o NCA, nos períodos matutino e vespertino, sendo ultrapassado no período noturno (Tabela 2). 
Figura 2 - Avaliação ambiental em P1

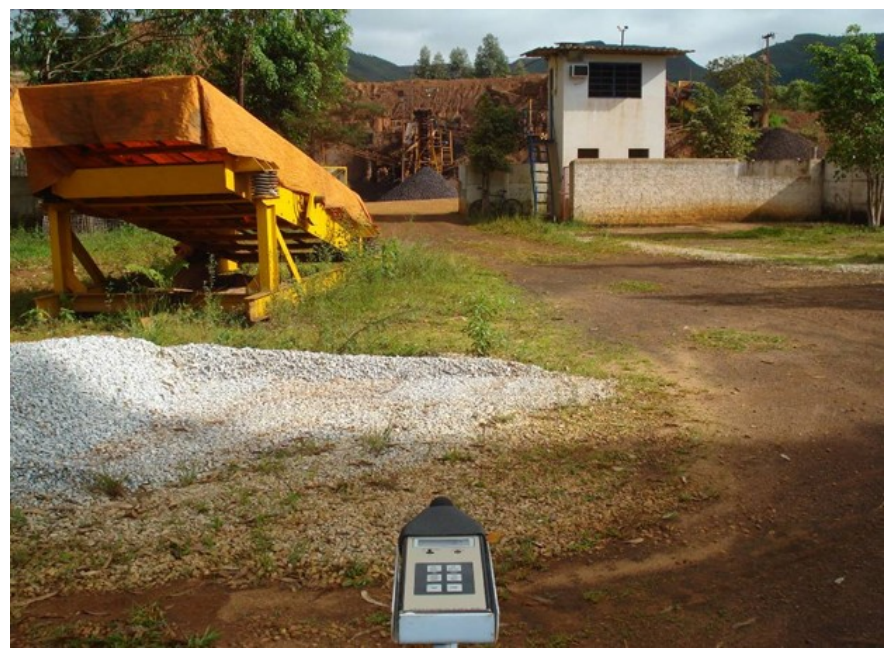

Fonte: Autoria própria (2017).

Os resultados explicitaram a necessidade de melhorias no Sistema de Gestão de Segurança do Trabalho (SGST) para o ambiente interno da empresa com resultados superiores ao NCA no período noturno. As propostas incluem a avaliação quanto às possibilidades de enclausuramento, ou até mesmo mudança de layout do britador. Além disso, recomenda-se a realização de manutenção preventiva e corretiva.

Tabela 2 - Resultado das avaliações no ambiente interno da mina (P1)

\begin{tabular}{cccc}
\hline \multicolumn{4}{c}{ PONTO 1 - Interno da Mina } \\
\hline Horário & LAeq & NCA & Análise do Resultado \\
$08: 38$ & 68,7 & 70,0 & LAeq $<$ NCA \\
$08: 43$ & 66,5 & 70,0 & LAeq $<$ NCA \\
$08: 48$ & 67,3 & 70,0 & LAeq $<$ NCA \\
& & VESPERTINO & \\
Horário & LAeq & NCA & Análise do Resultado \\
13:35 & 59,3 & 70,0 & LAeq $<$ NCA \\
13:40 & 55,5 & 70,0 & LAeq $<$ NCA \\
13:45 & 59,7 & 70,0 & LAeq $<$ NCA \\
& & NOTURNO & \\
Horário & LAeq & NCA & Análise do Resultado \\
19:00 & 66,2 & 60,0 & LAeq $>$ NCA \\
19:05 & 64,1 & 60,0 & LAeq $>$ NCA \\
19:10 & 65,7 & 60,0 & LAeq $>$ NCA \\
\hline
\end{tabular}

Fonte: Autoria própria (2017).

No entanto, no Ponto 2 (P2), que encontra-se em torno de $150 \mathrm{~m}$ da fonte geradora, o monitoramento ambiental (Figura 3) mostrou que o nível equivalente de ruído ficou abaixo do NCA, em todos os períodos avaliados (Tabela 3). 
Figura 3 - Foto do Ponto 2

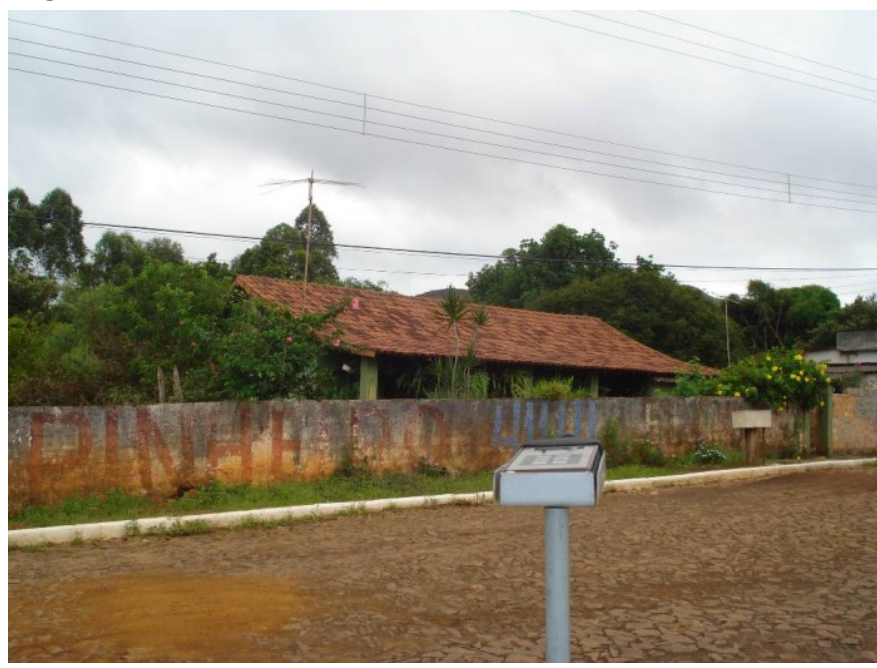

Fonte: Autoria própria (2017).

Tabela 3 - Resultado das avaliações no ambiente externo da mina, comunidade

\begin{tabular}{cccc}
\hline \multicolumn{4}{c}{ PONTO 2 Externo da Mina } \\
\hline Dorário & LAeq & NCA & Análise do Resultado \\
$09: 30$ & 53,5 & 70,0 & LAeq $<$ NCA \\
$09: 35$ & 52,1 & 70,0 & LAeq $<$ NCA \\
$09: 40$ & 52,7 & 70,0 & LAeq $<$ NCA \\
& & VESPERTINO & \\
Horário & LAeq & NCA & Análise do Resultado \\
14:13 & 56,6 & 70,0 & LAeq $<$ NCA \\
14:18 & 53,5 & 70,0 & LAeq $<$ NCA \\
14:23 & 53,2 & 70,0 & LAeq $<$ NCA \\
& & NOTURNO & \\
Horário & LAeq & NCA & Análise do Resultado \\
19:30 & 50,7 & 60,0 & LAeq $<$ NCA \\
19:35 & 53,1 & 60,0 & LAeq $<$ NCA \\
19:40 & 55,3 & 60,0 & LAeq $<$ NCA
\end{tabular}

Fonte: Autoria própria (2017).

Os achados desse estudo no ponto 2 (Tabela 3) mostraram NCA satisfatório em todas as medições, porém, insuficientes para garantir a inexistência de desconforto para população em seu entorno conforme a avaliação de percepção dos entrevistados. Com relação às queixas decorrentes do ruído no local de trabalho, o incômodo foi relatado em (100\%), seguido de intolerável $(50 \%)$ e irritante (80\%), dor de cabeça (40\%), diminuição da audição (20\%), alteração do sono $(13,5 \%)$, zumbido $(60 \%)$ e tontura $(30 \%)$, respectivamente (Figura 4$)$. 
Figura 4 - Principais queixas dos entrevistados

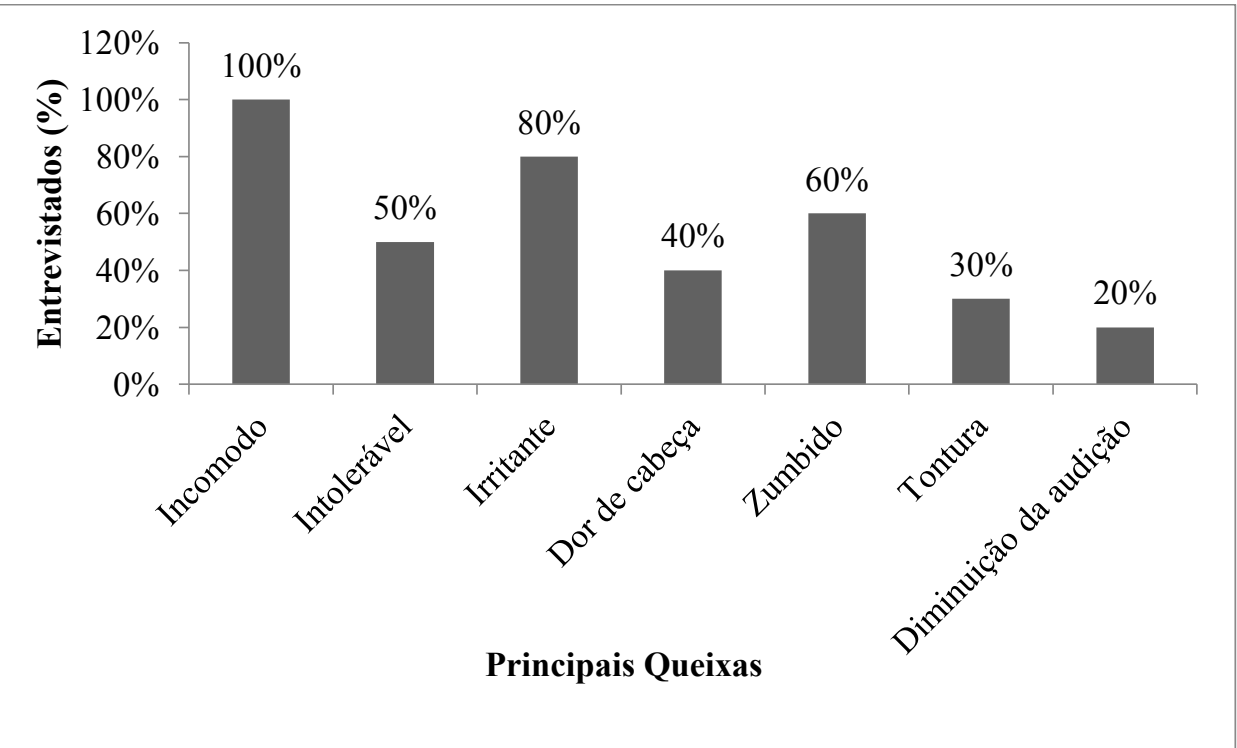

Fonte: Autoria própria (2017).

As condições desconfortáveis indicadas pelos entrevistados mostram-se como um indicador preoucupante. Vale ressaltar que a associação entre a sensibilidade ao ruído ambiente e as doenças cardíacas e a mortalidade cardiovascular entre adultos é reconhecida na ciência da medicina humana (KRAWCZAK et al, 2016). Estudos epidemiológicos desenvolvidos por Babisch (2006) permitiram detectar um acréscimo da mortalidade devido a doenças coronárias em indivíduos sensíveis ao ruído, principalmente entre as mulheres.

A exposição prolongada ao ruído classificado como incomodo ou intolerável (Figura 4) pode causar dores de cabeça, cansaço e elevação da pressão arterial podendo o indivíduo exposto sofrer infarto. Outros aspectos inerentes às possibilidades do adoecimento individual, o que ocorre é que o barulho prolongado causa uma reação de estresse e o sistema nervoso autônomo (aquele que regula os níveis de adrenalina) está mais estimulado (KZAWCZAK et al, 2016). O estimulo frequente causado pelo ruído, mantem a pressão arterial mais elevada, aumenta a resistência à insulina (deixando os níveis de glicose mais altos), contribuindo para o processo de aterosclerose (BABISCH, 2006).

Além disso, mostra-se importante considerar a possibilidade quanto à ocorrência da presbiacusia, considerada a perda da audição, observada com a idade (VAN BORSEL e BAECK, 2013). Viegas et al. (2015) ponderam que realmente a questão fisiológica integra o processo do envelhecimento humano com alterações no sistema auditivo,basicamente por processos de calcificação e perda de elasticidade das fibras do ouvido interno. Este processo de presbiacusia pode ser acelerado por exposição a ruídos em ambiente de trabalho, por ototoxidez e, também, pelo próprio ruído urbano (FUESS e LORENZI, 2003).

Quanto ao sintoma de dor de cabeça indicado por $40 \%$ dos entrevistados (Figura 4) sugeriu relação com a cefaléia tensional que é o tipo de dor de cabeça mais comum na população. Em geral, a disfunção orgânica acomete mais as mulheres que os homens e o estresse pode ser o fator desencadeador das crises (SALVAGNI et al., 2015). Nesse caso específico, infere-se que o ruído possa ser 
considerado como um agente contributivo causador desse distúrbio orgânico e recomenda-se que a população seja submetida a avaliação clínica criteriosa.

O zumbido no ouvido relatado por $60 \%$ dos entrevistados. Conforme, Holdefer et al., (2010) normalmente esse sintoma não retrata condições graves e surge em caso de resfriado ou após exposição prolongada a barulhos muito intensos, desaparecendo sem tratamento específico ao final de algumas horas. Todavia, o zumbido pode ser causado por infecções ou tumores auditivos e, por isso, estes sintomas também demandam análise clínica para consolidação do diagnóstico (ZANNIN et al., 2002).

A tontura relatada por $30 \%$ dos entrevistados (Figura 4) é uma patologia que faz com que o indivíduo tenha uma falsa sensação de movimento do ambiente que o rodeia, ou de si mesmo; além dessa péssima sensação, a tontura também causa desequilíbrio e sensação de enjoo (SÖHSTEN et al., 2016). Os achados dos estudos desenvolvidos por Bittar e Sohsten (2015) em pacientes com tontura mostram relação intrínseca com a exposição à poluição sonora. Novamente aí, reforça-se a necessidade quanto à potencialização dos mecanismos de proteção da população em relação ao ruído.

Em relação à percepção dos entrevistados quanto à sensação de perda de audição é comum em indivíduos expostos aos ambientes ruidosos. Araújo (2002) relatam que o ruído poder lesar considerável extensão das vias auditivas, desde a membrana timpânica até regiões do sistema nervoso central. Duarte et al. (2015) informam que as células auditivas ciliadas externas são particularmente sensíveis a pressões sonoras altas e prolongadas e podem ser dizimadas, resultando em déficit permanente na capacidade auditiva e nesse estudo a redução da capacidade auditiva foi relatada por $20 \%$ dos entrevistados (Figura 4).

A poluição sonora se destaca como um problema de saúde pública acarretando distúrbios físicos e psicológicos (LÜHNING et al., 2016). A Constituição Federal (CF/88) estabelece a competência administrativa, em matéria ambiental. Portanto, os problemas relativos aos níveis excessivos de ruídos estão incluídos entre os sujeitos ao controle da poluição ambiental, cuja normatização e estabelecimento de padrões compatíveis com o meio ambiente equilibrado são determinados pelo Conselho Nacional do Meio Ambiente (CONAMA).

Além disso, em função dos frequentes estudos acerca das consequências maléficas da poluição sonora sobre o organismo humano e da enorme quantidade de fontes causadoras de poluição sonora, esta vem sendo interpretada como crime de acordo com o artigo 54 da Lei 9.605/98 (BRASIL, 1998) que trata dos Crimes Ambientais. Nesse sentido, compete aos empreendimentos econômicos buscar alternativas para atenuar as condições desconfortáveis para a comunidade em seu entorno. Verifica-se aí a necessidade de aperfeiçoar as medidas de contenção da poluição, tal como, o plantio de árvores como estratégia para isolar as fontes de emissão de barulho da comunidade exposta através da Secretaria do Meio Ambiente do município. Entretanto, cabe também ressaltar a responsabilidade individual para a redução do barulho excessivo também dentro de suas casas, tal como, o uso inadequado de aparelhos sonoros (televisão, rádio, etc.). Destaca-se que qualquer tipo de ruído abusivo originário de uma propriedade particular, que venha a tirar a paz e 
o sossego dos vizinhos, o seu proprietário poderá sofrer as restrições estabelecidas pela sobredita legislação.

\section{CONCLUSÕES}

A poluição sonora é uma forma de agressão ambiental que é frequentemente negligenciada até em níveis muito prejudiciais. Esse tipo de poluição, ao contrário das outras, não deixa resíduo, existindo apenas no momento em que é produzida. Em decorrência disso, são formas de poluição consideradas menos perigosas. No entanto, sabe-se que a exposição repetida a estas formas de agressão pode produzir efeitos crônicos e irreversíveis, prejudicando a saúde e as relações sociais.

Com este estudo, pode-se fazer uma análise crítica dos resultados, através de medições do ruído ambiental gerado nessa mineradora, fundamental para se determinar uma solução de tratamento acústico mais indicado e eficiente. Para tanto, a solução de tratamento acústico deve-se levar em conta várias condições, conforme preconiza a ABNT 10152/00.

Com base nos resultados do diagnóstico realizado, o nível de ruído avaliado em ambiente externo da mineradora, ficou abaixo do NCA em todos os períodos avaliados, não influenciando assim na qualidade de vida de uma população que vive nas adjacências da mineradora.

Portanto, mesmo diagnosticado nesta pesquisa que o ruído gerado na mineradora não ultrapassa os limites determinados pela ABNT 10151/00, vale ressaltar que a poluição sonora deve ser vista como algo agressivo. O ruído é um poluente invisível que, lentamente, vai agredindo os indivíduos, causando-lhes danos tanto auditivo como em todo o organismo. 


\title{
EVALUATION OF ENVIRONMENTAL NOISE IN A MINING COMPANY
}

\begin{abstract}
The synchronization between industrial activities and business management are essential conditions for achieving economic control with a reduction of the destabilizing factors of human life and adverse repercussions on the other biotic components. In this sense, meeting the safety and health needs of workers and the population is intrinsically related. The importance of monitoring environmental risks is understood. For this reason, we sought to identify the noise-related impacts produced by a mining enterprise. The results obtained were then compared with the provisions of ABNT 10151/2000 and the recommendations of ISO / 1996. It was verified that the working conditions in the internal environment of the mine presented standard operating dynamics, however, with limits of unfavorable hearing conditions (noisy environments). Therefore, objective measures of control are allowed for workers, such as: enclosures, casings, barriers, insertion of attenuators. In the external environment of the mine, the noise generated was below the Auditory Comfort Level (NCA) in all evaluated periods. However, it was found that residents in the vicinity of the project presented complaints related to noise pollution. From this perspective, it is recommended to take full advantage of natural obstacles by investing in the maintenance of native vegetation.
\end{abstract}

KEYWORDS: Mining. Environmental noise. Workplace safety. Mitigation. 


\section{REFERÊNCIAS}

ANDRADE, D. C. (2008). Economia e meio ambiente: Aspectos teóricos e metodológicos nas visões neoclássica e da economia ecológica. Leituras de Economia Política, 14:1-31.

ARAÚJO, S. A. (2002). Perda auditiva induzida pelo ruído em trabalhadores de metalúrgica. Revista Brasileira de Otorrinolaringologia, 68(1), 47-52. crossref

ABNT (ASSOCIAÇÃO BRASILEIRA DE NORMAS TÉCNICAS). NBR 14724: Informação e Documentação - Trabalhos acadêmicos - Apresentação. Rio de Janeiro: ABNT, 2001.

ABNT (ASSOCIAÇÃO BRASILEIRA DE NORMAS TÉCNICAS). NBR 10151: Acústica Avaliação do ruído em áreas habitadas, visando o conforto da comunidade Procedimento. Rio de Janeiro: ABNT, 2000.

ABNT (ASSOCIAÇÃO BRASILEIRA DE NORMAS TÉCNICAS). NBR 10152: Nível de ruído para conforto acústico. Rio de Janeiro: ABNT, 2000.

$\mathrm{BABISCH}, \mathrm{W}$. Transportation noise and cardiovascular risk: updated review and synthesis of epidemiological studies indicate that the evidence has increased.

Noise \& Health. 2006, Vol. 8, pp. 1-29.

BELLUSCI, S. M. Doenças profissionais ou do trabalho. 6ạ Edição, São Paulo: Editora SENAC, 2012.

BENTO COELHO, J. L. Noise in cities and the role of the acoustician. International Journal of Acoustics and Vibration, v. 14, p. Editorial, 2009.

BITTAR, R. S. M.; SOHSTEN, E. M. D. Características Clínicas de Pacientes com Tontura Postural Perceptual Persistente. Brazilian Journal of Otorhinolaryngology (Online), v. 81, p. 276-282, 2015. crossref

BRASIL. Lei 9.605/98: Dispõe sobre as sanções penais e administrativas derivadas da lei de crimes ambientais, condutas e atividades lesivas ao meio ambiente (Lei de Crimes Ambientais).1998.

BRASIL. CONAMA. Resolução 001/90, de 08 de março de 1990. Dispõe sobre critérios e padrões de emissão de ruídos, das atividades industriais. Disponível em: http://www./mma.gov.br/port/conama/index.cfm. Acesso em: 08 out. 2016. 
BRASIL. Ministério do Trabalho. Limite de tolerância. Portaria 3214 de 08 de junho de 1978 - NR 15 - anexo 11.

CAIXETA, R. M.; RIBEIRO, D. T.; COSTA, J. F. C. L.; MACHADO, P. L. Multiple Random Walk Simulation: A Fast Method to Map Grade Uncertainty with Large Datasets. Natural Resources Research (New York, N.Y.), v. 40, p. 1-20, 2016.

CANDIA, R. C.; HENNIES, W. T.; Almeida, I. G.; AZEVEDO, R. C.; ELGUERA, J. F. S. Análisis de accidentes fatales en la industria minera. Boletín Geológico y Minero, v. 1, p. 57-68, 2010.

COPASA (Companhia de Saneamento de Minas Gerais). Disponível em: <www.copasa.com.br>. Acesso em: 13 jul. 2016.

DELA COLETA, J. A. Fator humano, contribuições da psicologia do trabalho, atividades de prevenção. São Paulo, Editora Atlas, 1989.

DI GIULIO, G. M.; VASCONCELLOS, M. P. C.; GUNTHER, W. M. R.; RIBEIRO, H.; ASSUNÇÃO, J. V. Percepção de risco: um campo de interesse para a interface ambiente, saúde e sustentabilidade. Saúde e Sociedade (Online), v. 24, p. 12171231, 2015. crossref

DUARTE, A. S. M.; NG, R. T. Y.; CARVALHO, G. M.; GUIMARAES, A. C.; PINHEIRO, L. A. M.;COSTA, E. A.; GUSMÃO, R. J. High levels of sound pressure: acoustic reflex thresholds and auditory complaints of workers with noise exposure. Brazilian Journal of Otorhinolaryngology (Online), v. 81, p. 374-383, 2015. crossref

FRANCO, J. M. V. Contributos do Estudo da Exposição ao Ruído Ocupacional Análise de uma Central Termoeléctrica. Dissertação (Mestrado) Engenharia de Segurança e higiene Ocupacionais. Faculdade de Engenharia da Universidade do Porto, 2010.

FUESS, V. L. R.; LORENZI, M. C. Disfonia em Professores do Ensino Municipal: Prevalência e Fatores de risco. Revista Brasileira de Otorrinolaringologia, São Paulo, v. 69, n.6, p. 807-812, 2003. crossref

GOLDNER, L. G.; NASCIMENTO, A. M.; FREITAS, I. M. D. P. Análise do aeroporto Salgado Filho como pólo gerador de viagens. Journal of Transport Literature, v. 8, p. 229-249, 2014. crossref

HARGER M. R.; BARBOSA-BRANCO, A. Efeitos auditivos decorrentes da exposição ocupacional ao ruído em trabalhadores de marmorarias no Distrito Federal. Rev Assoc Med Bras. 2004; 50 (4):396-9. crossref 
HOLDEFER, L.; DE OLIVEIRA, C. A. C. P.; VENOSA, A. R. Sucesso no tratamento do zumbido com terapia em grupo. Brazilian Journal of Otorhinolaryngology (Impresso), v. 76, p. 102-106, 2010. crossref

IBGE - INSTITUTO BRASILEIRO DE GEOGRAFIA E ESTATÍ́STICA, 2000. Resultado dos Dados Preliminares do Censo - 2000. Disponível:<www.ibge.gov.br/cidade>, Acesso: 10/11/2016.

KÄHARI K.; ZACHAU, G.; EKLÖF, M.; SANDSJÖ, L.; MÖLLER, C. Assessment of hearing and hearing disorders in rock/jazz musicians. Int J Audiol. 2003;42:27988. crossref

KRAWCZAK, E. M.; MINUZZI, L.; Hidalgo, M. P.; FREY, B. N. Do changes in subjective sleep and biological rhythms predict worsening in postpartum depressive symptoms? A prospective study across the perinatal period. Archives of Women's Mental Health, v. 26, p. 1-8, 2016.

MAFIA, R.G.; ALFENAS, A.C.; FERREIRA, E. M.; BINOTI, D.H.B.B.; MAFIA, G.M.V.; MOUNTEER, A.H.. Root colonization and interaction among growth promoting rhizobacteria isolates and eucalypt species. Revista Árvore (Impresso), v. 33, p. 19, 2009. crossref

MAHENDRA P. K. V.; SRIDHAR, V. The relationship between noise frequency components and physical, physiological and psychological effects of industrial workers. Noise Health. 2008; 10(40): 90-8. crossref

LOBO SOARES, A. C.; COELHO, T. C. C.; COELHO, J. L.; BENTO . Análise da Paisagem Sonora de Quatro Parques Públicos Na Amazônia Brasileira. Acústica e Vibrações, v. 47, p. 3-10, 2015.

LÜHNING, A. E.; LOPES, Aaron Roberto de Mello ; Cachineski, Flávia ; Carvalho Tiago . Ethnomusicological Goals and Challenges in Brazil. The World of Music (Wilhelmshaven), v. 5, p. 23-53, 2016.

RABINOWITZ, P. M.; GALUSHA, D.; DIXON-ERNST, C.; SLADE, M. D.; CULLEN, M. R. Do ambient noise exposure levels predict hearing loss in a modern industrial cohort? Occup Environ Med. 2007; 64(1):53-9. crossref

SALVAGNI, C.; GIARETTA, V. M. de A.; POSSO, M. B. S. Ruído na área de recepção e limpeza de produtos para a saúde de um centro de material e esterilização. Revista SOBECC (São Paulo), v. 20, p. 157-162, 2015. crossref 
SANTOS, D. V. de C.; DE FREITAS, I. M. D. P. Medidas de Mobilidade Urbana Sustentável (MMUS): propostas para o licenciamento de Polos Geradores de Viagens. Transportes (Rio de Janeiro), v. 22, p. 11-22, 2014.

SANTOS, U. P. S. Exposição a Ruído: Avaliação de Riscos, Danos à Saúde e Prevenção. In.: MATOS, M. P. et al. Ruído Riscos e Prevenção. São Paulo: Ed. Hucitec, 1994. p. 35-38.

SETHURAJAN, M.; HUGUENOT, D.; LENS, P.; HORN, A. H.; ARIMURA, L. H. F.; HULLEBUSCH, E. V. Fractionation and leachability of heavy metals from aged and recent $\mathrm{Zn}$ metallurgical leach residues from the Três Marias zinc plant (Minas Gerais, Brazil). Environmental Science and Pollution Research International, v. 1, p. 10-17, 2016. crossref

SILVA, C. H.; LASCHEFSKI, K. Conflitos ambientais no entorno do Parque Estadual da Serra do Brigadeiro (PESB): Agricultura Familiar e mineração de bauxita no Município de Miradouro. Por Extenso: Boletim de Pesquisa do Programa de PósGraduação em Extensão Rural, v. 4, p. 20, 2012.

SILVA, J. P. S. Impactos ambientais causados por mineração. Revista Espaço da Sophia. Ano 01, n. 08, Nov. 2007.

SÖHSTEN, E.; BITTAR, R. S. M.; STAAB, J. P. Posturographic profile of patients with persistent postural-perceptual dizziness on the sensory organization test. Journal of vestibular research equilibrium \& orientation, v. 26, p. 319-326, 2016.

SUTER, A. H. Construction noise: exposure, effects, and the potential for remediation; a review and analysis. AlHA J (Fairfax, Va). 2002; 63(6):768-89.

crossref

VAN BORSEL, J.; BAECK, H. E. The voice in transsexuals. Revista de Logopedia, Foniatría y Audiología (Ed. Impresa), v. 34, p. 20-48, 2013.

VIEGAS, F.; VIEGAS, D.; BAECK, H. E. Frequency Measurement of Vowel Formants Produced by Brazilian Children Aged Between 4 and 8 Years. Journal of Voice, v. 29, p. 292-298, 2015. crossref

WHO (World Health Organization). Night Noise Guidelines for Europe. Copenhagen: s.n., 2009.

ZANNIN, P.H.T. Acústica Ambiental. Paraná: UFPR, 2004. 
ZANNIN, PHT; CALIXTO, A.; DINIZ, F.B.; FERREIRA, J.A.; SCHULI, R.B. Incômodo causado pelo ruído urbano à população de Curitiba, PR. Rev. Saúde Pública 2002; 36 (4): 521-4. crossref

Recebido: 23 jan. 2017

Aprovado: 12 set. 2017

DOI: 10.3895/gi.v13n2.5339

Como citar:

QUEIROZ, M. T. A. et al. Avaliação do ruído ambiental em uma mineradora. R. Gest. Industr., Ponta

Grossa, v. 13, n. 2, p. 198-214, jun./ago. 2017. Disponível em: <https://periodicos.utfpr.edu.br/rgi>. Acesso em: XXX.

Correspondência:

Marluce Teixeira Andrade Queiroz

Av. Presidente Tancredo de Almeida Neves, 3500, Bairro Universitário, Coronel Fabriciano - Vale do Aço Minas Gerais, Brasil.

Direito autoral: Este artigo está licenciado sob os termos da Licença Creative Commons-Atribuição 4.0 Internacional.

(c) (1) 